\title{
Paucity of secondary synaptic clefts in a case of congenital myasthenia with multiple contractures: ultrastructural morphology of a developmental disorder
}

\author{
LME SMIT,${ }^{*}$ FGI JENNEKENS $\uparrow \dagger$ H VELDMAN,$\dagger$ PG BARTH $\ddagger$
}

From the Department of Child Neurology, University Hospital of the Free University, Amsterdam, * Laboratory for Neuromuscular Diseases, Department of Neurology, University Hospital of the State University. Utrecht, $\dagger$ Department of Child Neurology, Academic Medical Centre, Amsterdam, $\ddagger$ the Netherlands

SUMMARY A new form of congenital myasthenia is described. An infant whose foetal movements during pregnancy had been weak presented at birth with muscle weakness and multiple contractures of the lower limbs. The clinical course was characterised by myasthenic crises during febrile illnesses. Neurophysiological studies demonstrated a decremental response at 2-3 HZ stimulation; this effect was reversed by edrophonium iv. At the age of eight months, a biopsy from the soleus muscle showed a predominance of type I fibres and variation in fibre diameters was slightly increased. Ultrastructural studies of the motor endplates revealed a marked reduction of postsynaptic membrane lengths with paucity of secondary clefts. Signs of focal degeneration were absent. The picture was reminiscent of foetal neuromuscular junctions. A developmental disorder of the postsynaptic membrane was considered to be the basic cause of the neuromuscular transmission defect.

Myasthenia is considered to be congenital if it presents before the age of two years in patients without circulating antibodies against acetylcholine receptors (AchR). Congenital myasthenia has been shown to be a heterogeneous condition. ${ }^{2}$ Originally, two types were distinguished on the basis of clinical presentation: ${ }^{3}$ (1) familial infantile myasthenia in siblings with episodes of severe respiratory and feeding difficulties, lack of obvious extraocular muscle involvement and a tendency for spontaneous remission; (2) congenital myasthenia sensu stricti, manifest at birth or even during foetal life, usually involving extra-ocular muscles, together with persistent clinical symptoms.

Electrophysiological, ultrastructural and his-

\footnotetext{
Address for reprint requests: Drs LME Smit, Laboratorium voor Neuromusculaire Ziekten, Kliniek voor Neurologie, Academisch Ziekenhuis, Postbus 16250, 3500 CG Utrecht, Nederland.
}

Received 4 January 1984 and in revised form 29 March 1984 Accepted 1 April 1984 tochemical studies carried out in the last five years, have shown that various defects of pre- and postsynaptic elements underlie true congenital myasthenia. To date, three different syndromes have been reported: (a) a deficiency of endplate acetylcholinesterase, small nerve terminals and reduced acetylcholine release $;{ }^{4}$ (b) putative defect of acetylcholine resynthesis or mobilisation; ${ }^{5}$ and (c) a putative defect of the acetylcholine-induced ion channel. 6

In 1980 , we described a patient with congenital myasthenia and arthrogryposis congenita.? The results of ultrastructural studies of motor endplates in this child are the subject of this paper. We shall present evidence to support the hypothesis that the transmission defect in this patient is due to a hitherto undescribed developmental disorder of the postsynaptic membrane.

\section{Case history}

The boy was born as the first child of young, healthy and non-consanguineous parents. Foetal movements during 
pregnancy had been weak. Delivery was spontaneous with cephalic presentation. Birth weight was $3500 \mathrm{~g}$. Postnatally, he showed mild respiratory distress with insufficient chest expansion, a weak cry, generalised muscle hypotonia and weakness, in particular witli reference to sucking and swallowing. There were multiple contractures of the lower limbs. Gavage feeding was initially necessary. During the first months, the boy's mental development was normal, but there was a marked delay in motor milestones, together with progressive deformation, resulting in a funnel chest, scoliosis and subluxation of the hips.

An upper respiratory tract infection at the age of five months provoked bilateral ptosis, facial diplegia and tetraplegia. Myasthenia was diagnosed by means of a positive edrophonium test. Treatment was initiated with pyridostigmine bromide and this resulted in marked improvement of muscle strength. This treatment arrested progression of the orthopaedic abnormalities and facilitated motor development.

Immunological investigations showed that antibodies reactive to skeletal muscle and to the AchR were absent in the sera of mother and child.

Electromyography showed a 55\% decrease in amplitude of action potentials during stimulation at the rate of 3 per second, and a progressive diminution of action potentials during stimulation at a rate of 30 per second. This effect was reversed by intravenous administration of $1 \mathrm{mg}$ edrophonium. The percentage of the decremental response was calculated as the reduction in amplitude of the response to the fifth stimulus, compared to the amplitude of the response to the first stimulus.

\section{Materials and methods}

\section{Patient material}

At the age of eight months, a surgical correction of the talipes equinovarus was performed and a soleus muscle biopsy was taken for study of neuromuscular junctions.

\section{Control material}

Control soleus muscle specimens were obtained from two other 8 month-old infants with talipes equinovarus, in whom physical examination and routine histochemical investigations of muscles had shown no signs of neuromuscular disorders. These two controls, and the patient, had been subjected to prolonged immobilisation with plaster bandages prior to surgical correction. An intercostal muscle biopsy from another 8 month-old infant, with coarctation of the aorta, served as a third control.

\section{Methods}

One part of each muscle biopsy was immediately frozen in isopentane, cooled in liquid nitrogen and stored at $-85^{\circ} \mathrm{C}$ until use. Transverse cryostat sections measuring $8 \mu \mathrm{m}$, were used for routine histological and histochemical staining. Maximum/minor diameters were used for measurement of fibre size. Endplates and intramuscular nerves were stained in $50 \mu \mathrm{m}$ cryostat sections according to Pestronk and Drachmann. ${ }^{8}$ Other muscle strips, tied to a wooden stick or clamped in a forceps to prevent contraction, were fixed for three hours in $2 \%$ glutaratiehycte solus: tion, buffered with $0 \cdot 1 \mathrm{M}$ cacodylate $(\mathrm{pH} 7 \cdot 3)$ containing
$10 \mathrm{mM} \mathrm{CaCl}_{2}$.

Endplate regions were located, as described by Engel," by cholinesterase staining according to the Koelle and Friedenwald method. ${ }^{10}$ These parts were postfixed for two hours in $1 \%$ osmium tetroxide solution, dehydrated and embedded in epon, as in routine EM procedure. Motor endplates were identified in $1 \mu \mathrm{m}$ sections stained with toluidine blue. Ultrathin sections were prepared from selected regions and contrasted with uranyl acetate followed by lead citrate and viewed in the electronmicroscope (Zeiss 109, Carl Zeiss, Oberkochen, FRG). Each neuromuscular junction, so observed, was photographed. A neuromuscular junction was defined as a nerve terminal with synaptic vesicles and the postsynaptic area of folds and clefts associated with it. When more than one neuromuscular junction occurred in an endplate section, each was analysed separately. A total of 56 neuromuscular junctions were studied in the patient, 61 neuromuscular junctions in control soleus muscles and 28 in the control intercostal muscle.

\section{Morphometric analysis}

A computerised Image Analysis System (Videoplan, Kontron Messgeräte, West Germany) was used for measuring the terminal axon area, the postsynaptic area and the preand postsynaptic membrane lengths of each neuromuscular junction. We defined the postsynaptic area as the sarcoplasm between the postsynaptic membrane and the contractile elements. Synaptic vesicle diameter and synaptic clefe width were measured by hand with a measuring magnifier (Polaron, MM15, Watford, Hertfordshire, GB). The presynaptic cleft length was not measured separately, but was considered to be equal to the length of the presynaptic membrane.

Statistical analysis of the quantitative data was made by means of the non-parametric Mann-Whitney U test.

\section{Results}

\section{Light microscopy}

In cross sections, a number of disseminated small angular fibres were seen. Histograms were constructed (fig 1) according to fibre size and type, as judged from the myosine ATPase reaction. " Nearly all the fibres were between 10 and $20 \mu \mathrm{m}$ in diameter. Fibres outside this range were used to calculate the atrophy-hypertrophy $(\mathrm{A}-\mathrm{H})$ factors. In our patient, these factors were: Type 1, 38-50 and Type $2,32-65$. A-H factors in the two soleus controls were: Type 1, 16-7, Type 2, 7-17 and Type 1, 10 45 , Type 2, 21-8, respectively. No structural changes were seen in fibres. Some fascicles consisted of $90 \%$ Type 1 fibres, while others showed a slight Type 2 predominance. The overall Type 1/Type 2 ratio was $1: 3$ (soleus controls $1: 2.5$ and 1.4) Cholinesterase staining was positive. In serial sections, no more than one innervation site was seen per muscle fibre. Combined staining of motor end- plates and intramuscular nerves showed no evidence of terminal sprouting. 


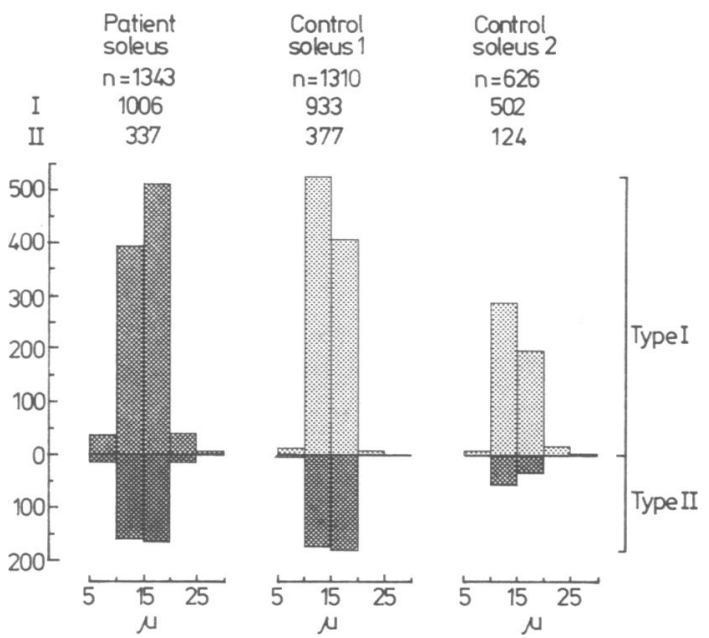

Fig 1 The patient muscle contains more small and more large fibres than controls.

\section{Electron microscopy}

The terminal axons had a normal appearance and a normal Schwann cell covering. Some terminals contained large numbers of mitochondria and some of these were artificially swollen. The synaptic vesicles were normal in apparance and distribution. There was no focal widening of the primary synaptic cleft.
In the synaptic clefts, a monolayer of basal lamina material was visible.

Striking abnormalities were found in the postsynaptic parts of the neuromuscular junctions. The postsynaptic membranes were abnormally straight with few, if any, infoldings. The secondary clefts, when transversely sectioned, were circular in appearance, suggesting a tube-like shape. They were often positioned parallel to the primary synaptic cleft in contrast to the normal perpendicular position of the clefts in controls. The postsynaptic membranes contained many pinocytotic vesicles and in some places the membranes were coated. Because of the paucity of secondary clefts and their parallel position to the primary synaptic cleft, the postsynaptic areas were very small and consisted of shallow zones between the postsynaptic membrane and the contractile elements. In these areas, the mitochondria, tubular system, smooth and rough sarcoplasmic reticulum and glycogen distribution appeared normal.

\section{Morphometric analysis}

The mean terminal axon area and the presynaptic membrane length were not significantly different from the soleus and intercostal control values. There was a marked variability in the terminal axon surface. The synaptic vesicles were similar to those in controls and measured $400-500 \AA$ in diameter. The

terminal axon area

presynaptic membrane length

2 postsynaptic membrane length

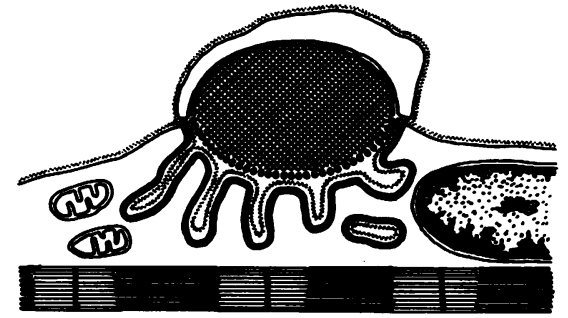

Results:

muscle biopsy

number of neuromuscular junctions

mean terminal axon area $\left(\mu \mathrm{m}^{2} \pm \mathrm{sD}\right)$

mean presynaptic membrane length $(\mu \mathrm{m} \pm \mathrm{SD})$

mean postsynaptic membrane length $(\mu \mathrm{m} \pm \mathrm{SD})$

ratio $\frac{\text { postsynaptic membrane length }}{\text { presynaptic membrane length }}( \pm \mathrm{SO})$

infoldings $/ 10 \mu \mathrm{m}$ primary synaptic cleft ( $\pm \mathrm{SO}$ )

\begin{tabular}{|c|c|c|c|}
\hline patient & control 1 & control 2 & control 3 \\
\hline soleus & soleus & soleus & intercostal \\
56 & 29 & 32 & 28 \\
$5.77 \pm 4.9$ & $6.15 \pm 5.1$ & $3.18 \pm 2.4$ & $3.97 \pm 3.8$ \\
$5.56 \pm 2.8$ & $6.11 \pm 2.8$ & $4.25 \pm 1.6$ & $5.87 \pm 3.1$ \\
$15.33 \pm 9.2$ & $31.98 \pm 14.6$ & $26.97 \pm 10.8$ & $24.40 \pm 11.1$ \\
$2.95 \pm 1.55$ & $5.95 \pm 2.7$ & $6.9 \pm 3.3$ & $5.0 \pm 2.6$ \\
$2.4 \pm 2.9$ & $17.0 \pm 5$ & $17.7 \pm 7$ & $15.3 \pm 5$
\end{tabular}

Fig 2 Morphometric analysis results. 

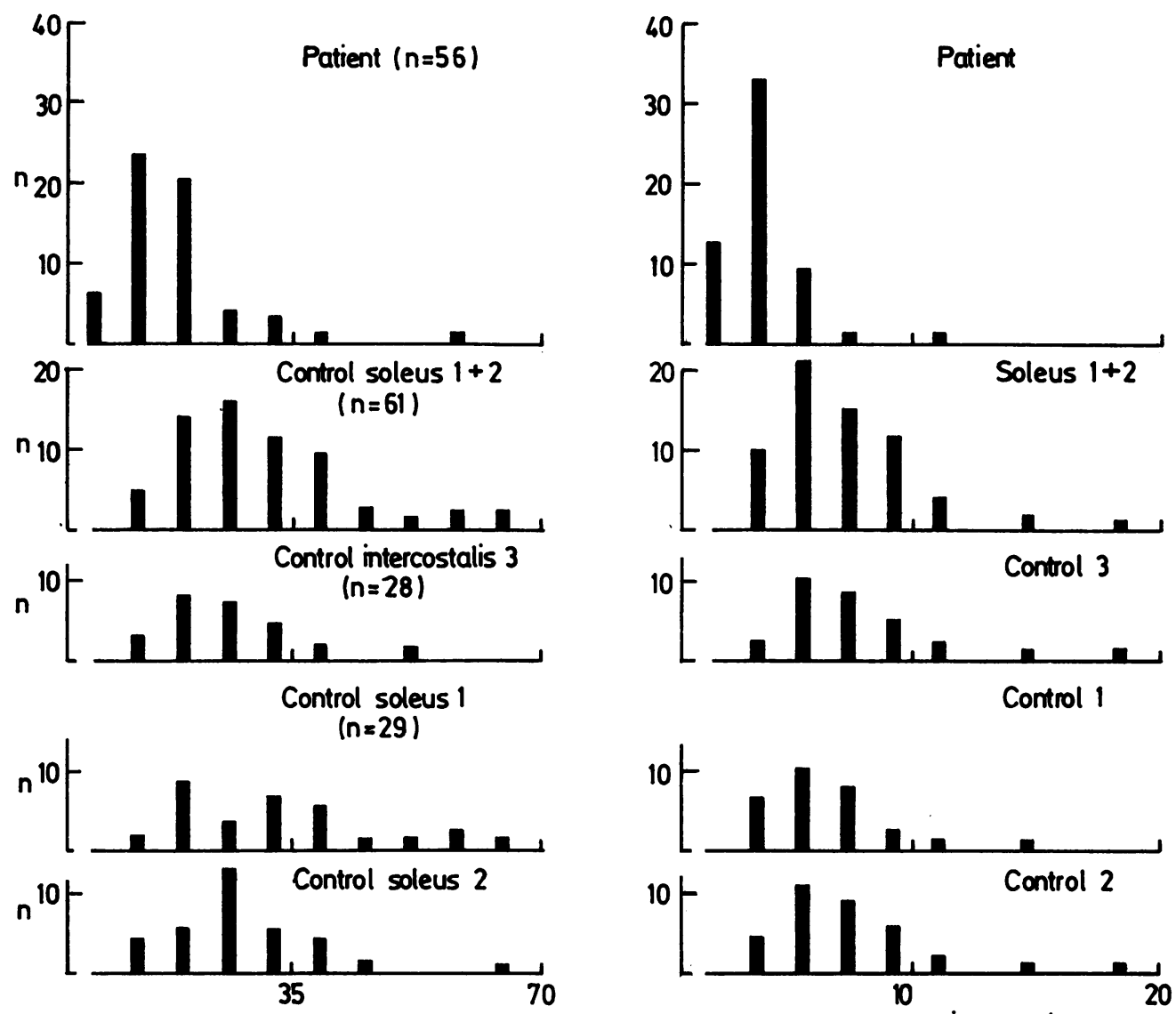

Postsynaptic membrane length

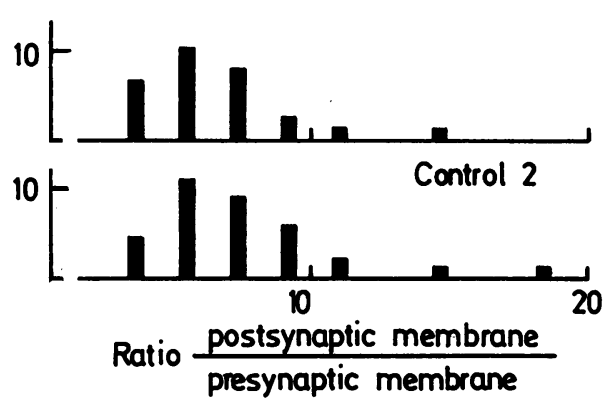

Fig 3 Morphometric analysis: histograms. There is a marked predominance of short postsynaptic membranes in the patient; the ratios have a different maximum. These data are compatible with a highly simplified postsynaptic membrane.

primary synaptic cleft length did not differ from controls and its width was normal.

The mean postsynaptic membrane length was about half that of the controls and when the ratio postsynaptic membrane/presynaptic membrane length was calculated per neuromuscular junction, the difference was striking $(p<0.001)$. As there was no apparent difference in the length of the primary clefts, the difference in postsynaptic membrane length could be attributed mainly to the diminished length of the secondary clefts.

In our patient, the average number of infoldings per $10 \mu \mathrm{m}$ was calculated to be $2 \cdot 4$, whereas in controls there were 17 infoldings per $10 \mu \mathrm{m}$ length. The sevenfold reduction in the number of infoldings, together with the fact that there was only a two-tothreefold reduction in postsynaptic membrane length, implies that the secondary clefts are longer.

\section{Discussion}

The clinical picture included multiple contractures and variable muscle weakness from birth. Prior to treatment, there was no complete remission, and febrile illnesses exacerbated the muscle weakness. Electro-physiological investigations, showing a decreased response on repetitive stimulationreversible by intravenous administration of edrophonium-were conclusive for the diagnosis of myasthenia. 

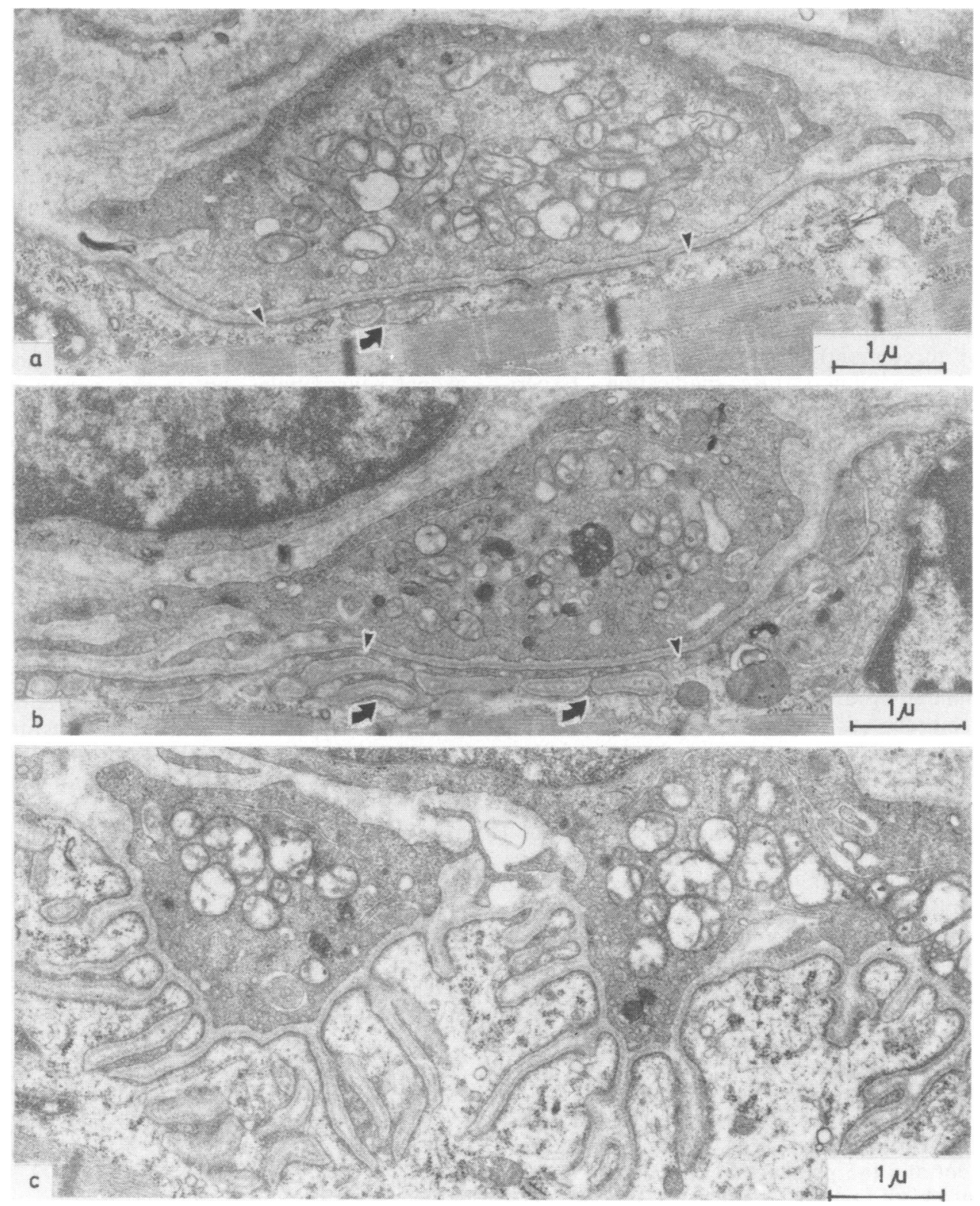

Fig $4(a, b)$ The neuromuscular junctions in the patient muscle have practically no postsynaptic membrane infoldings (arrowheads). The secondary clefts have a tube-like appearance (arrows). (c): Control soleus neuromuscular junctions, showing normal perpendicular postsynaptic membrane infoldings.

As no antibodies to acetylcholine receptor protein could be detected in the sera of mother and child, a diagnosis of autoimmune myasthenia gravis could almost definitely be excluded. The negative family history and the presence of ptosis at birth, were compatible with the diagnosis congenital myasthenia.

Light microscopy of the soleus muscle biopsy showed a slightly increased variability in diameter of the muscle fibres. The $\mathrm{A}-\mathrm{H}$ factors were greater 
than in the controls. We have no explanation for this finding. The normal fibre type distribution indicated that the neuromuscular transmission disorder had permitted a normal maturation as expressed in fibre type differentiation.

The ultrastructural findings with regard to the postsynaptic membrane, were highly abnormal. In our case, this membrane had fewer infoldings than controls and the secondary clefts were not arranged perpendicularly but almost parallel to the postsynaptic membrane. In fact, the secondary clefts did not look like clefts at all, but were more tube-like and circular in cross sections.

In the three months prior to biopsy, our patient received anticholine-sterase medication. Experimental studies have shown that structural changes may be induced by anticholinesterase drugs. The ultra-structural findings in the present case differed completely, however, from the degenerative alterations due to longterm anticholinesterase therapy, and also from the changes in membrane-bound organelles after acute treatment with a low dose of neostigmine. ${ }^{12} 13$

Postsynaptic ultrastructural defects have been reported in two other forms of congenital myasthenia: degenerative changes were described in the slow-channel syndrome, ${ }^{\circ}$ and focal degeneration and membraneous networks which communicate with the synaptic space, in the endplatecholinesterase deficiency syndrome. ${ }^{4}$ In our patient signs of degeneration, such as widening of the primary or secondary synaptic clefts, with focal cytoplasmic degeneration, are totally absent.

Reduction of the postsynaptic membrane length and alterations in the shape of the secondary clefts are common findings in auto-immune myasthenia, but these changes are associated with degeneration and regeneration. ${ }^{14}$ The neuromuscular junctions in our patient resemble human foetal endplates at 16-20 weeks gestation. At 16 weeks, the postsynaptic membrane begins to fold and a few clearly defined secondary clefts become apparent. At 20 weeks, the clefts become more numerous and deeper. ${ }^{15} 16$ The clinical signs of decreased foetal movement and the presence of congenital contractures at birth are compatible with an early neuromuscular transmission disturbance, due to a defect in the development of the neuromuscular junction.

Factors which have been considered to influence the endplate development are: motor activity, nerve conduction, axonal transport and the metabolic and neuro-physiologic properties of the AChR channel complex. ${ }^{17}$ Experiments with neonatal rats have shown that muscle immobilisation by tenotomy of the tibialis anterior muscle, results in considerable atrophy of the muscles whilst folding of the postsynaptic membrane occurs normally. ${ }^{18}$ Prolonged nerve blockade with local anaesthetics, resulting in a cessation of impulse-evoked release of $\mathrm{ACh}$ in the newborn rat, has no effect on the induction folds in the postsynaptic membrane. ${ }^{19}$ Folding of the postsynaptic membrane is, however, prevented by local application of cholchicine or vinblastine around the innervating nerve. These substances are known to inhibit the fast phase of axoplasmic transport in the nerve fibres and they are known to cause temporary disappearance of the axonal neurotubules and increase of neurofilaments. ${ }^{20}$ During the subsequent 3 weeks, there is a gradual reappearance of the axonal neurotubules, but in this period the postsynaptic membrane remains unfolded."

Recent publications point to the fact that postsynaptic events also bear some relationship to the infoldings of the postsynaptic membrane..$^{22}$ Before the nerve-muscle contact has been established, AChR is distributed along the total surface of the muscle fibre. At the time that endplate formation takes place, there is a change in distribution and gating properties of the AChR channel complex. At the same time, infolding of the postsynaptic membrane occurs. Bio-electrical and bio-chemical properties of the postsynaptic membrane are, therefore, related in time to the molecular architecture of this membrane. Hence there is evidence that presynaptic factors, as well as postsynaptic membrane properties, play a role in the folding of the postsynaptic membrane. New techniques of receptor-marking have demonstrated that the acetylcholine receptors on the postsynaptic membrane are located in the direct environment of the infoldings. ${ }^{23}$ Underdevelopment of the postsynaptic membrane, with paucity of secondary clefts, is likely to result in a decrease in the number of AChRs, with subsequent inadequate neuromuscular transmission.

We thank AM Stadhouders, G Egberink, SL Visser, RA Hoogland, G Wieneke and SM Dierks-Mallett for their help in preparing this manuscript.

\section{References}

' Engel AG. Morphologic and immunopathologic findings in myasthenia gravis and in congenital myasthenic syndromes. $J$ Neurol Neurosurg Psychiatry 1980;43:577-89.

${ }^{2}$ Vincent A, Cull-Candy SG, Newsom-Davis J, Trautmann A, Molenaar PC, Polak RL. Congenital myasthenia: end-plate acetylcholine receptors and electro-physiology in five cases. Muscle Nerve 1981;4:306-18.

${ }^{3}$ Fenichel GM. Clinical Syndromes of myasthenia in infancy and childhood. Arch Neurol 1978;35:97-103. 
${ }^{4}$ Engel AG, Lambert EH, Gomez MR. A new myasthenic syndrome with end-plate acetylcholinesterase deficiency, small nerve terminals and reduced acetylcholine release. Ann Neurol 1977;1:315-30.

${ }^{5}$ Hart Z, Sahashi K, Lambert EH, Engel AG, Lindstrom JM. A congenital, familial myasthenic syndrome caused by a presynaptic defect of transmitter resynthesis or mobilization. (Abstract) Neurology (Minneap) 1979;29:556-7.

- Engel AG, Lambert EH, Mulder DM, Torres CF, Sahashi K, Bertorini TE, Whitaker JN. A newly recognized congenital myasthenic syndrome attributed to a prolonged open time of the acetylcholine-induced ion channel. Ann Neurol 1982;11:553-69.

' Smit LME, Barth PG. Arthrogryposis multiplex congenita due to congenital myasthenia. Dev Med Child Neur 1980;22:371-4.

${ }^{8}$ Pestronk A, Drachman DB. A new stain for quantitative measurement of sprouting at neuromuscular junctions. Muscle Nerve 1978;1:70-4.

${ }^{9}$ Engel AG. Locating motor end plates for electron microscopy. Mayo Clin Proc 1970;45:450-4.

${ }^{10}$ Koelle GB, Friedenwald JS. A histochemical method for localizing cholinesterase activity. Proc Soc Exp Biol Med 1949;70:617-22.

"Brook MH, Engel WK. The histographic analysis of human muscle biopsies with regard to fiber types. Neurology (Minneap) 1969;19:378-93.

12 Engel AG, Lambert EH, Tetsuji S. Study of long-term anti-choline-sterase therapy. Neurology (Minneap) 1973;23: 1273-81.

${ }^{13}$ Hudson CS, Rash JE, Tiedt TN, Albuquerque EX. Neostigmine-induced alterations at the mammalian neuromuscular junction. II Ultrastructure. J Pharmacol Exp Ther 1978;205:340-56.
${ }^{14}$ Zacks SI, Bauer WC, Blumberg JM. The fine structure of the myasthenic neuromuscular junction. $J$ Neuropath Exp Neurol 1962;21:335-47.

${ }^{15}$ Fidzianska A. Human ontogenesis. Development of the human neuromuscular junction. $J$ Neuropath Exp Neurol 1980;39:606-15.

${ }^{16}$ Juntunen J, Teräväinen $\mathrm{H}$. Structural development of myoneural junctions in the human embryo. Histochemie 1972;32:107-12.

17 Juntunen J. Induction of the postsynaptic membrane. Medical Biology 1974;52:164-169.

18 Juntunen J. Morphogenesis of the myoneural junctions after immobilization of the muscle in the rat. $Z$ Anat Entwicklungsgesch 1973;143:1-12.

${ }^{19}$ Robert ED, Oester YT. Absence of supersensitivity to acetylcholine in innervated muscle subjected to a prolonged pharmacological block.J Pharmacol Exp Ther 1970; 174: 133-40.

${ }^{20}$ Ochs S. Fast axoplasmic transport of materials in mammalian nerve and its integrative role. Ann NY Acad Sci 1972; 193:43-58.

${ }^{21}$ Juntunen J. Effects of colchinine and vinblastine on neurotubules of the sciatic nerve and cholinesterase in the developing myoneural junction of the rat. Z Zellforsch 1973; 142: 193-204.

${ }^{22}$ Michler A, Sakmann B. Receptor stability and channel conversion in the subsynaptic membrane of the developing mammalian neuromuscular junction. Developm Biology 1980;80:1-17.

${ }^{23}$ Engel AG, Lindstrom JM, Lambert EH, Lennon VA. Ultrastructural localization of the acetylcholine receptor in myasthenia gravis and its experimental autoimmune model. Neurology (Minneap) 1977;27:30715. 